Type of the Paper (Article, Review, Communication, etc.)

\title{
Problems with Abstract Observers and Advantages of a Model- Centric Paradigm
}

Mick Ashby 1,2,3,*

1 The Cybernetics Society: http://cybsoc.org
2 The W. Ross Ashby Digital Archive: $\underline{\text { ttp://rossashby.info }}$
3 The American Society for Cybernetics: $\underline{\text { https://asc-cybernetics.org }}$
* Correspondence: ethics@ashby.de

\begin{abstract}
Since 1974, when Heinz von Foerster made the distinction between "the cybernetics of observed systems" as first-order cybernetics $(1 \mathrm{oC})$ and "the cybernetics of observing systems" as second-order cybernetics (2oC), cybernetics has been dominated by this observer-centric paradigm that he claimed cannot be extended meaningfully to a third-order. Rather than attempting to extend his paradigm, we derive an alternative, model-centric paradigm from first principles of regulation that naturally extends to three orders, where the third order is ethical regulation. We thus consider a type of regulator that requires a third model and a third observer: If the third model is a model of acceptable (ethical) situations, then a third observer is a necessary element of the system's "conscience" that prevents or detects any violations of the model of ethical situations. In this paradigm, the cybernetics of systems that are designed to exhibit ethical behaviour can be characterized as third-order cybernetics $(3 \mathrm{oC})$. By being able to extend the paradigm to include ethical systems, the model-centric paradigm brings clarity and utility that is not possible using the philosopher-friendly observer-centric paradigm and its under-specified (abstract) observers. Finally new definitions for cybernetics are proposed that clearly differentiate between the science and the philosophy, of cybernetics.
\end{abstract}

Keywords: ethical robots; ethical artificial intelligence; ethical cybernetics; third-order cybernetics; philosophy of cybernetics; empathy; sapientocracy

\section{Introduction}

In 1948, Norbert Wiener defined cybernetics as "the scientific study of control and communication in the animal and the machine" [1]. This defines a scope for cybernetics that includes all types of regulators and communicators. And if we accept that philosophy is not science, then Wiener's original definition of cybernetics excludes any philosophical aspects.

In 1974, Heinz von Foerster [2] made the distinction between "the cybernetics of observed systems" as first-order cybernetics $(1 \mathrm{oC})$ and "the cybernetics of observing systems" as second-order cybernetics $(2 \mathrm{oC})$. Since 1974, the cybernetics community has been dominated by this observer-centric paradigm.

Although he might have intended it purely as a distinction between two approaches to performing cybernetics, it has been widely generalized as a model for understanding different types of systems. Thus, endowing meaning to terms like first-order system and second-order system, as illustrated by Ranulph Glanville "The question arises about differences in how we observe a system that is first or second order." [3].

However, von Foerster's definitions use the terms "observed" and "observing" without being more specific. Of course, there will be an underlying purpose behind any observations, but the abstract conceptualization of a pure observer has no purpose ex- 
cept observing, which is like a person who is meditating so deeply with open unfocussed gazing eyes that they neither react to what they observe nor make memories of it.

When the observer has a well-defined purpose, "observer" ceases to be the best description of them. For example, if the purpose of an observer is to create a model of the system that is observed, then the best name for them is "modeler", or if they are observing the system to ensure that it breaks no laws, then the names "auditor" or "conscience" are more accurate and useful descriptions than "observer". And because there are an infinite number of possible reasons why an observer might observe a system, the term "observing" is completely ambiguous with respect to purpose, which renders it deficient in clarity and utility.

Because the expression "observing systems" is devoid of actual purpose, von Foerster's definition of $20 \mathrm{C}$ could be rewritten as "the cybernetics of $\mathrm{X}$, where $\mathrm{X}$ is any activity that requires observations to be made". Stated in this way, the extreme ambiguity of his definitions becomes more obvious, and Margaret Mead's "cybernetics of cybernetics" is only one of many possible valid interpretations. And to equate $2 \mathrm{oC}$ with a particular special case interpretation is not a scientific consideration of all possibilities.

So, when von Foerster introduced $2 \mathrm{oC}$ as "the cybernetics of observing systems" it had multiple possible interpretations that went beyond Wiener's precisely defined "scientific study of control and communication" to include just about anything, such as "thinking about thinking" and "understanding understanding", which have been a consideration of philosophers dating back to the ancient Greeks and continues today in philosophy departments around the world. This rescoping of the definition of cybernetics was effectively an open invitation to any philosophers who self-identified as cyberneticians to participate in the cybernetic discourse. And von Foerster's brilliant pivot of Wiener's field of cybernetics from a science to being a hybrid of science and philosophy only enhanced the profound intellectual inclusiveness that is not enjoyed by any other science.

Consequentially, 1974 can be regarded as the year of the schism of what was originally Wiener's science of cybernetics into two very different communities, which can be characterized as "the science of cybernetics" and "the philosophy of cybernetics". This schism has been as significant as the distinction between $10 \mathrm{C}$ and $20 \mathrm{C}$. Whereas Kline [4] and Scott [5] considered it and other disunities from a historical perspective, this paper is more concerned with the limitations of von Foerster's observer-centric paradigm.

\section{Diversity and Dichotomy}

Although this paper takes a position that must be contrasted with von Foerster's interpretation of $2 \mathrm{oC}$ as the cybernetics of cybernetics or second-order observation, which we characterize as being philosophical, it is not intended to suggest that his approach was unscientific or that philosophical aspects are completely unscientific, less worthy, or that they should be excluded from cybernetics, but rather that cybernetics embodies both scientific and philosophical approaches, and that the more philosophical interpretation of the meanings of $1 \mathrm{oC}$ and $2 \mathrm{oC}$ as increasing orders of observation has arguably supressed the equally valid possible scientific interpretations of $1 \mathrm{oC}$ and $2 \mathrm{oC}$ as increasing orders of regulation. The use of these characterizations of one as being philosophical and the other as being scientific is not intended to deny that there is a significant complementary overlap between both approaches.

This paper explores the implications of the second interpretation, and reveals how considering increasing orders of regulation produces valuable results, which have practical implications for artificial intelligence (AI), robotics, and society that cannot be derived from the more philosophical observer-centric interpretation ${ }^{1}$.

With the benefit of hindsight, some might claim that the new results that are described in this paper can be derived using the observer-centric interpretation of orders of cybernetics as orders of observation, but this is only now made possible with the new insights that were rendered thinkable by completely abandoning the use of abstract (under-specified) observers, which 
Just as the boundary of the system being considered by a cybernetician can be decided arbitrarily as seems most useful, the distinction of whether a system contains an observer $(2 \mathrm{oC})$ or not $(1 \mathrm{oC})$ is quite arbitrary because there are many other equally valid criteria that can be used to slice the field of cybernetics into two parts. Yet when a cybernetician is deciding what the boundaries are of what she will consider to be the system being studied or its environment, she strives to maximize clarity and utility.

However, this is not true of von Foerster's observer-centric $1 \mathrm{oC} / 2 \mathrm{oC}$ paradigm. By arbitrarily introducing observers that were not present in Wiener's definition, clarity and utility have not been maximized. Although asserting observers into existence might seem intuitively unproblematic, it introduced unstated assumptions, and it is not constructed from first principles.

Suddenly, everyone felt entitled to offer their own alternative definitions of $1 \mathrm{oC}$ and $2 \mathrm{oC}$, as described by Stuart Umpleby [6]. Table 1 is based on a table from Umpleby, but with entries added for Wiener and Mead to provide more context for this discussion.

Table 1: Definitions of cybernetics

\begin{tabular}{|c|c|c|}
\hline Author & First-Order Cybernetics & Second-Order Cybernetics \\
\hline Wiener & \multicolumn{2}{|c|}{ The scientific study of control and communication } \\
\hline Mead & Cybernetics & The cybernetics of cybernetics \\
\hline Von Foerster & $\begin{array}{l}\text { The cybernetics of observed } \\
\text { systems }\end{array}$ & The cybernetics of observing systems \\
\hline Pask & The purpose of a model & The purpose of the modeler \\
\hline Valera & Controlled systems & Autonomous system \\
\hline Umpleby & $\begin{array}{c}\text { Interaction among the varia- } \\
\text { bles in a system }\end{array}$ & $\begin{array}{l}\text { Interaction between observer and } \\
\text { observed }\end{array}$ \\
\hline Umpleby & Theories of social systems & $\begin{array}{c}\text { Theories of the interaction between } \\
\text { ideas and society }\end{array}$ \\
\hline
\end{tabular}

This diversity of definitions reflects that while some cyberneticians remained focussed on the practical scientific study of real systems, others entered the realms of philosophy, for example, exploring the circularity of Margaret Mead's "cybernetics of cybernetics" and radical constructivism, just as in 1955, on page 4814 of his journal, Ross Ashby had reflected on the circularity of his brain studying "the brain":

"For some time, I have had the hunch that there is a profound circularity in science. Since I have to use a mammalian brain to think with, I am likely to find in the world only what it lets me find. Ultimately, as the 'world as I see it' is analysed, I shall find simply that I am looking, as it were, at the underside of my own cortex." [7]

From the point-of-view of cybernetics as a science, interpreting second-order cybernetics as "the cybernetics of cybernetics" rather than "the cybernetics of reflexive systems", of which "the cybernetics of cybernetics" is merely a special case, can be viewed as a decision that established a philosophy of cybernetics that has dominated the cybernetic narrative and has attenuated the scientific study of the cybernetics of reflexive systems.

This paper's criticism of von Foerster's $1 \mathrm{oC} / 2 \mathrm{oC}$ paradigm is not intended to suggest that it has not been useful. Even if it has weaknesses, it made an important distinction that helped cybernetics develop into what it is now. But after more than 45 years as the dominant paradigm in cybernetics, we suggest that it has probably contributed pret-

was necessary to extend cybernetics' Overton window. Despite the fact that von Foerster gave his 1974 "Cybernetics of cybernetics" BCL publication [2] the subtitle "Or, the control of control and the communication of communication", the uncomfortable fact is that, for over 45 years, some combination of groupthink and hero-worship appears to have effectively blinded the cybernetics community to the (now suddenly "obvious") equally valid possible interpretation of orders of cybernetics as being orders of regulation. 
ty much all that it ever can, and that it is time to find a new paradigm that can bring cybernetics firmly back into the realms of science.

The practitioners of the philosophy of cybernetics have certainly added an incredible richness to the field of cybernetics, but it has been at the cost of making cybernetics appear to be less scientific. And it is clear that topics such as "thinking about thinking", "understanding understanding", and radical constructivism represent significant shifts towards philosophy and away from cybernetics as a science.

But we are facing so many serious problems and challenges globally, socially, ecologically, economically, and technologically, that what we desperately need is better cybernetics, not better philosophy.

\section{The Possibility of Third-Order Cybernetics}

In a 1990 interview, von Foerster categorically asserted that there is no possibility of the existence of a meaningful definition of third-order cybernetics:

"... it would not create anything new, because by ascending into 'second-order,' as Aristotle would say, one has stepped into the circle that closes upon itself. One has stepped into the domain of concepts that apply to themselves." [8].

Ranulph Glanville went further to claim that a third-order system cannot exist because it collapses into being equivalent to a first-order system:

"The question arises about differences in how we observe a system that is first or second order. If we talk about (observe) a second order system in the cool manner in which we are used to talking about such things, we might claim we are, in effect, creating a third order system, which we can, however, collapse into a first order one (because the observing is of the first order type, no matter what type the observed system is)."[3].

It is important to note that von Foerster's argument is philosophical rather than scientific, and Glanville is clearly arguing from within von Foerster's observer-centric paradigm.

Despite these claims by von Foerster and Glanville that defining third-order cybernetics would not create anything new, many people have attempted to do exactly that, by extrapolating from von Foerster's definitions of $1 \mathrm{oC}$ and $2 \mathrm{oC}$ to define $30 \mathrm{oC}$. The result has been various competing intelligent and creative proposals for possible definitions of $30 \mathrm{C}$ that are merely plausible. None have had arguments that were sufficiently compelling to gain widespread acceptance.

\section{The Model-Centric Cybernetics Paradigm}

Rather than trying to extrapolate from von Foerster's observer-centric definitions, we shall construct an alternative paradigm from first principles, seeking to maximize clarity, precision, and utility, completely avoiding any reliance on the problematic concept of abstract observers, and above all making the new paradigm rigorously scientific such that Wiener might have given it his approval.

If we start with Wiener's original definition of cybernetics as "the scientific study of control and communication", from the word "control" we can infer that it includes all types of regulators, $\mathrm{R}$, and we can assume that they have a purpose, $\mathrm{P}$, to regulate an entity that we can refer to as the regulated system, $\mathrm{S}$.

For completeness, we must also consider Wiener's use of the word "communication". If a communication has any effect on the recipient, we can regard it as a special type of control. And if it has no effect on the recipient or if the communication reaches no receiver, then it is still equivalent to an act of control but it corresponds to the special case of a non-action.

Here it is worth highlighting that control and communication lie on the continuum of degrees of influence. Communication has only a probabilistic influence on the regulated system, and control has a deterministic influence (i.e. its probability of effective influence $=1$ ). 


\section{First-Order Regulators: Simple Regulators}

Let us define a first-order cybernetic regulator, $R_{1}$, that has a purpose, $P_{1}$. In order for it to be effective, $\mathrm{R}_{1}$ must have a model, $\mathrm{M}_{1}$, of the regulated system, $\mathrm{S}$. This is a direct consequence of the Conant-Ashby good regulator theorem, which proved that "every good regulator of a system must be a model of that system" [9]. We know that $\mathrm{M}_{1}$ is a model of $\mathrm{S}$, but the scope and precision of $\mathrm{M}_{1}$ are very much determined by $\mathrm{P}_{1}$.

The effect of purpose on the scope and detail of the model can be illustrated by the fact that in a conceptual model of a pig brain, the complexity and level of detail must be considerably higher for a researching neurologist to be effective than is required by a butcher. They both require models of the same system, but it is the difference in their purposes that requires very different actual models.

Next, because a fundamental characteristic of a model is that it requires observations as inputs, the model not only brings into existence the need for an observer, $\mathrm{O}_{1}$, to exist in $R_{1}$, but that observer is not an unconstrained abstract observer that might observe anything (or everything). The inputs of $\mathrm{M}_{1}$ define very precisely what information $\mathrm{O}_{1}$ must collect. Therefore, in contrast to von Voerster's observers, $\mathrm{O}_{1}$ is scientifically well-defined.

However, having a purpose, $\mathrm{P}_{1}$, appropriate model, $\mathrm{M}_{1}$, and our well-defined observer, $\mathrm{O}_{1}$, is insufficient to realize the functionality of an effective regulator. We must introduce an entity that is capable of making appropriate decisions to select the best actions (or communications) to achieve effective regulation. This decision-making unit must embody some kind of intelligence that could be biological, algorithmic, or baked into the design by the intelligence of the designer. Because we require clean definitions, this decision-making intelligence cannot be attributed to the observer being an intelligent observer (which would be a dangerous antrhopomorphization), it must be identified as a separate component of $R_{1}$, which we shall name $I_{1}$. Finally, the regulator requires a control channel, $\mathrm{C}_{1}$, that transmits the selected action or communication to the regulated system. These necessary elements of a cybernetic regulator are illustrated in Figure 1.

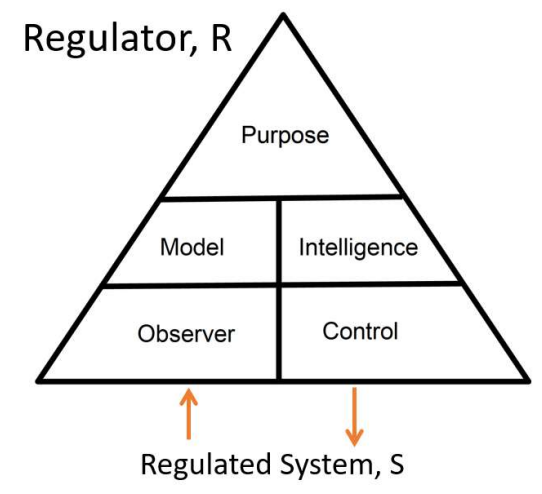

Figure 1: A cybernetic regulator

Now we have a complete definition that a first-order cybernetic regulator, $\mathrm{R}_{1}$, consists of a purpose $\mathrm{P}_{1}$, model, $\mathrm{M}_{1}$, observer, $\mathrm{O}_{1}$, decision-making intelligence, $\mathrm{I}_{1}$, and control channel, $\mathrm{C}_{1}$. And Heinz would surely not hesitate to confirm that such a regulator conforms to his understanding of a first-order regulator that can be designed using $10 \mathrm{C}$.

It is worth highlighting that we refer to von Foerster's $1 \mathrm{oC} / 2 \mathrm{oC}$ paradigm as being observer-centric because the observer is given a primary role in his definitions, as if everything revolves around the cybernetician and his observations, which has possibly cognitively biased cyberneticians to think of observers as being intelligent observers, like themselves. But in the model-centric paradigm, the observer is arguably the least important element in the regulator because it serves simply as a data source to obtain in- 
formation to feed the model. So, it is fair to say that $\mathrm{O}_{1}$ is to $\mathrm{M}_{1}$ and $\mathrm{I}_{1}$ as a keyboard is to a program and a computer. Observers and keyboards are necessary, but they are neither the most interesting nor the most important parts.

\section{Second-Order Regulators: Reflexive Regulators}

Our next step is to consider reflexive regulators that have to take themselves into account, such as the U.S. Federal Reserve's Federal Open Market Committee (FOMC), which generally meets every six weeks to decide whether to change the U.S. central bank's base interest rate. In addition to needing predictive economic models in $\mathrm{M}_{1}$, it can only be effective if it also has self-knowledge about how long it is until the next meeting, which is its next scheduled opportunity to make another interest rate change. If the FOMC decided that the next meeting would be in six months instead of the normal sixweek cadence, this self-knowledge must be taken into account and affect their decision making and some parameters of the economic simulations that they run. The variable "time-till-next-meeting" does not need to be predicted probabilistically because it is $100 \%$ under the FOMC's direct control, which is to say that it belongs to the variety of the FOMC rather than to the $\mathrm{M}_{1}$ model of the regulated economic system.

Another example is that a two-armed robot only requires a $10 \mathrm{C}$ regulator to control the arms to perform simple tasks. But implementing reflexivity could mean that if it detects that one arm is non-functional, it could try to achieve its tasks using just the one arm that works.

So, a second-order cybernetic regulator, $R_{2}$, not only includes all of $R_{1}$, but it must also have a self-referential purpose, $\mathrm{P}_{2}$, of taking itself into account, and maintain a second model, $\mathrm{M}_{2}$, which captures key aspects about itself. This model requires selfobservations that must be performed by a second observer, $\mathrm{O}_{2}$, that is well-defined by the needs of the second model. And it must also include some degree of decisionmaking intelligence, $\mathrm{I}_{2}$, and a control channel, $\mathrm{C}_{2}$.

Another example is that because of our constant subconscious self-monitoring of our health and vitality, if we find ourselves in a situation where we need to jump over an object, our $\mathrm{M}_{2}$ model can, at any moment, provide a sense of self-knowledge of how high and how far we are confident of being able to jump. When we are ill or tired, knowing that we cannot jump a stream or a log could be important for our survival. The model of Newtonian physics that allows us to instinctively know how to jump effectively has been learned through trial and error, and is stored in our $\mathrm{M}_{1}$ model of how to interact effectively with our environment. So $\mathrm{I}_{2}$ uses $\mathrm{M}_{2}$ to provide potentially complex reflexive information to $I_{1}$ via channel $C_{2}$, which implies that in humans and animals, the nature of $\mathrm{C}_{2}$ is electrical.

If the $\mathrm{M}_{2}$ model can provide real-time information about what $\mathrm{R}$ is capable of in terms of possible actions and communications, then it is actually the source of R's knowledge of its own variety. Much has been made of the law of requisite variety [10], and people often talk of having requisite variety as if it were some sort of mystical deus ex machina, but the $\mathrm{M}_{2}$ model appears to be where information about possible variety of the regulator is stored.

Again, Heinz and second-order cyberneticians would surely agree that the regulator $R_{2}\left(R_{1}(S)\right.$ ) conforms to their concept of a second-order (reflexive) cybernetic regulator that can be designed using $2 \mathrm{oC}$, but cannot be constructed using just $1 \mathrm{oC}$.

In humans, there are fuzzy boundaries between models, like when someone is learning to drive a car with manual gear shifting. Initially they are very conscious of their hands, feet, pedals, gear-stick, and looking into mirrors. But with time and practice their ability to drive migrates into an instinctive subconscious ability. Thus, the possible biological optimizations and black-box nature of human minds create limits for being able to use human examples to confirm or illustrate the theory of discrete orders of regulation and models. However, these limitations do not apply to robots, where such distinctions will likely become very important. 
Of course, it can be argued that the $\mathrm{M}_{1}$ model could be extended to include all the reflexive information that we propose is encoded in the $\mathrm{M}_{2}$ model, but that is an implementation optimization that ignores that reflexivity is a significant and important difference that deserves to be treated separately. If an existing regulator is repurposed to regulate a different type of system, it would be disadvantageous if the original implementation did not clearly distinguish between the regulated system and the regulator. Self-knowledge is a different category of information from the probabilistic cause-effect information of the $\mathrm{M}_{1}$ model. For example, if we recognize that we do not have sufficient variety to control a system adequately, as humans, we can perform a strategic reflexive self-evaluation to identify which of our abilities we can improve to increase our quality (not quantity) of possible variety to improve the quality of our performance as a regulator, and such improvements are limited to variables that belong to the $\mathrm{M}_{2}$ model. And when we consider regulators such as AI and robots, the regulator must have a clear distinction between what is "self" that is subject to essentially deterministic direct control and things in its environment that are interacted with and require probabilistic predictions of their future states, values, positions, or behaviour. So, it is optimal to use $\mathrm{M}_{1}$ to make predictions about the regulated system, and use $\mathrm{M}_{2}$ to provide self-knowledge about available variety.

\section{Third-Order Regulators: Ethical Regulators}

Because we are exploring a model-centric paradigm, rather than an observer-centric paradigm, we are now ready to imagine a type of regulator, $R_{3}$ that includes all of $R_{2}$, but also requires a third type of model.

This is a key difference from previous approaches that have tried to extend von Foerster's observer-centric paradigm by adding a third observer on top of the existing two observers, which are maximally ambiguous, and can lead people into philosophical interpretations that ignore real systems.

But there is no problem adding a third model in the model-centric paradigm because although $\mathrm{R}_{3}$ can be regarded as a speculative extrapolation, it is building on top of our purposeful $\mathrm{R}_{2}$, which is defined more precisely and rigorously than von Foerster's 2oC.

If a third-order regulator, $\mathrm{R}_{3}$ has a purpose $\mathrm{P}_{3}$ of constraining $\mathrm{R}_{2}\left(\mathrm{R}_{1}(\mathrm{~S})\right)$ to exhibit only ethically acceptable behaviour, then $\mathrm{R}_{3}$ must have a model, $\mathrm{M}_{3}$, of what constitutes acceptable (ethical) behaviour or outcomes. In addition, it also requires a third observer, $\mathrm{O}_{3}$, a third decision-making intelligence, $\mathrm{I}_{3}$, and a third control channel, $\mathrm{C}_{3}$, to realize what we can characterize as the system's "auditor", "conscience", or "sense of integrity" that prevents or detects any violations of the model of ethical outcomes by the regulator, $R$ that is the union of $R_{1}, R_{2}$, and $R_{3}$.

Whereas the purpose of $R_{1}$, is always simple effectiveness, and the purpose of $R_{2}$ is always reflexive effectiveness, the purpose of $R_{3}$ is always ethical effectiveness, and it is appropriate to regard them as increasing orders of regulation.

So, we have specified a third-order regulator, $R_{3}\left(R_{2}\left(R_{1}(S)\right)\right)$, as illustrated in Figure 2.

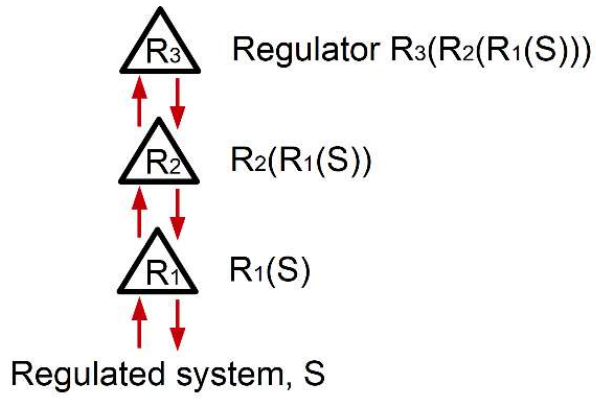

Figure 2: A Third-Order Regulator 
In such a regulator, its observer, $\mathrm{O}_{3}$, must observe the intended action, $\mathrm{A}$, before it is executed. This makes it possible for its decision-making intelligence, $\mathrm{I}_{3}$, to check whether the anticipated outcome of the action violates any imperatives or constraints in the $\mathrm{M}_{3}$ model of what is desirable, acceptable, or unacceptable, in which case I3 uses $\mathrm{C}_{3}$ to veto the action.

This is no different to the faculty that most (but not all) adults have that is running as a background process, constantly monitoring what we are about to say or do, and can kick in completely unexpectedly at any moment to stop us from saying or doing something that might be considered rude, hurtful, or otherwise socially unacceptable.

Throughout this paper, an ethical system is equated with one that is internally constrained to only exhibit behaviour that is defined as being acceptable in the culture within which the system exists. If we accept that a society's laws, regulations, and rules are its operational best approximation to that society's most important ethical principles, then what this paper refers to as an ethical regulator can effectively be interpreted as being a strictly law-abiding regulator.

In a more complex example, such as an autonomous robot, Isaac Asimov's First Law of Robotics: "A robot may not injure a human being or, through inaction, allow a human being to come to harm." [11] would require that inaction be treated as if it were a type of action that can be vetoed to force replanning to be performed to avoid a predicted unacceptable outcome of inaction. And even though it is generally impossible to get agreement on a definition of what is ethical or unethical, we can surely agree that systems such as robots and AI must be designed to be strictly and provably law-abiding.

In humans, the $\mathrm{R}_{3}$ regulation mechanism can be viewed as functioning at an executive level that controls motivational salience, with $\mathrm{O}_{3}$ monitoring both the intended action, A, and the anticipated outcome ${ }^{2}$ of performing $\mathrm{A}$, then based on the $\mathrm{M}_{3}$ model of the acceptability of outcomes, I3 can respond in three distinct ways:

- If the outcome is unacceptable, $\mathrm{I}_{3}$ uses control channel $\mathrm{C}_{3}$ to convey a veto alert to prevent the intention from being executed and thus force urgent replanning to be performed to avoid A.

- If the outcome is especially acceptable, such as achieving an important goal, money, or the object of an addiction, I3 uses $C_{3}$ to convey excitement for the anticipated outcome, and causes the intended action to be performed decisively with enthusiasm, without further delay.

- If the outcome is merely acceptable, I3 does nothing, which is an expression of indifference, which allows the intention to be acted upon consciously after some further delay, but without any enthusiasm.

It is clear that $\mathrm{I}_{3}$ is maximally utilizing the available $\mathrm{C}_{3}$ channel capacity by providing aversive, incentive, or neutral motivational salience as either negative feedback, positive feedback, or no feedback. And rather than sending a ternary signal with just the three simple values no/yes/don't-care, the signal could be a continuous linear value, such as a simple range from -1 to +1 or a vector.

In humans, this signal is not cognitive, it is a visceral feeling of fear, excitement, or indifference, and is therefore more likely to be transmitted using hormones or neurotransmitters rather than electrical signals.

We have all experienced that feeling when faced with a difficult decision, where we experience a pause in our conscious decision making, where we are effectively waiting to see if our subconscious has any objections to our currently preferred action. Many people refer to it as tuning into their gut instinct. We can understand it as subjecting our preferred action to the calculation $\mathrm{M}_{3}\left(\mathrm{M}_{1}\left(\mathrm{O}_{1}, \mathrm{~A}\right)\right)$ to see if it causes a positive or negative visceral response. A lack of visceral response leaves the conscious mind in control and able to rationally consider other possibilities or to initiate the action consciously.

2. Expressed simplistically, the distribution of probable outcomes is the result of computing $\mathrm{M}_{1}\left(\mathrm{O}_{1}, \mathrm{~A}\right)$. 
And neurological studies published in 2008 by Soon, Brass, Heinze, and Haynes have demonstrated that there is indeed a delay between the subconscious decision to act and the action being executed: "the outcome of a decision can be encoded in brain activity of prefrontal ${ }^{3}$ and parietal cortex up to $10 \mathrm{~s}$ before it enters awareness." [12].

Evolution (or intelligent design) would only create such a large delay between decision and action if it has significant advantages. Naturally, $\mathrm{M}_{3}$ must include probable injury or death of oneself as unacceptable outcomes, which makes the $R_{3}$ regulation mechanism a key advantage for survival. It also corresponds remarkably precisely with Ross Ashby's 1941 concept of a mechanism for adaptation, which he described on page 859 of his journal:

"A new idea. Suppose the cortex is a 'representation' of the environment, i.e. corresponding to events in the periphery (stimuli, reactions) there are events in the cortex. Nothing new in this idea. But suppose that the cortex is more 'sensitive', so that if the periphery is being driven out of its range of stability the corresponding variable in the cortex will break first, i.e. get outside its range of stability, and thus switch to another portion of the field (p. $\underline{817})$, and change partially to another organisation." [13]

He uses the term "stability" for what we refer to as "acceptability" and his concept of a "break" is equivalent to our concept of a "veto". Table 2 provides a phrase-byphrase translation of his description into the equivalent model-centric paradigm terminology that is used in this paper.

Table 2: Correspondence between Ross Ashby's 1941 mechanism for adaptation and the modelcentric paradigm

\begin{tabular}{|c|c|}
\hline $\begin{array}{c}\text { Ross Ashby's description of a } \\
\text { mechanism for adaptation }\end{array}$ & Model-centric paradigm translation \\
\hline $\begin{array}{c}\text { But suppose that the cortex is } \\
\text { more 'sensitive', }\end{array}$ & $\begin{array}{c}\text { But suppose that the outcome of an intention } \\
\text { can be predicted (by the M Model), }\end{array}$ \\
\hline $\begin{array}{c}\text { so that if the periphery is being } \\
\text { driven out of its range of stability }\end{array}$ & $\begin{array}{c}\text { so that if the intention is outside the range of } \\
\text { acceptability }\end{array}$ \\
\hline $\begin{array}{c}\text { the corresponding variable in the } \\
\text { cortex will break first, i.e. get out- } \\
\text { side its range of stability, }\end{array}$ & $\begin{array}{c}\text { the predicted outcome will violate the M3 model } \\
\text { i.e. get outside the range of acceptability) caus- } \\
\text { ing I to veto the execution of the intention }\end{array}$ \\
\hline $\begin{array}{c}\text { and thus switch to another por- } \\
\text { tion of the field, }\end{array}$ & $\begin{array}{c}\text { and thus force replanning to be performed to } \\
\text { explore alternatives, }\end{array}$ \\
\hline $\begin{array}{c}\text { and change partially to another } \\
\text { organisation. }\end{array}$ & $\begin{array}{c}\text { and exist in a world where the original intention } \\
\text { has not been executed and where the regulator } \\
\text { has possibly learned from the experience. }\end{array}$ \\
\hline
\end{tabular}

Whether a person's M3 model of acceptable outcomes extends to the protection of others, and not just the individual themselves, could depend on the presence of empathy, or perhaps the $\mathrm{M}_{3}$ model is actually the basis for empathy, with the emotions that are associated with empathy being caused by the $\mathrm{M}_{3}$ model making emotionally communicated acceptability predictions based on inputs from activated mirror neurons. This is credible since mirror neurons were shown to exist in the human inferior parietal cortex by Chong, Cunnington, Williams, Kanwisher, and Mattingley, in 2008, by using functional magnetic resonance imaging (fMRI) [14].

Our $\mathrm{M}_{3}$ models must develop during infancy and childhood and be considerably determined by our experiences, environment, and culture. So, it seems reasonable to assume that if a child witnesses suffering being inflicted upon others without negative consequences for the perpetrator, they are more likely to develop an $\mathrm{M}_{3}$ model where

3. The prefrontal cortex (PFC) has been implicated in executive functions such as decision making, planning, and moderating social behaviour. 
negative outcomes for others are not regarded as unacceptable and therefore, the child will become more likely to exhibit sociopathic and psychopathic behaviours that are likely to continue in adulthood ${ }^{4}$.

In machines, building an $R_{3}$ regulator into a system that is already reflexively effective has the potential to create AI and robots that don't just obey laws, but that have a synthetic sense of empathy. This could mean that they would not only realize that an action or situation is acceptable or unacceptable, but also that someone needs help.

Rather than every manufacturer trying to make each of their products ethically and empathically adequate, we speculate that perhaps it is possible to create a generic cybernetic level three AI supervisor that can be added as an executive controller to cybernetic level two $\mathrm{t}^{5} \mathrm{AI}$ or robots, with the sole purpose of constraining the product to behaving according to an appropriate ethical schema. It could also include an obligation to help people in need, as is required by Asimov's First Law of Robotics ${ }^{6}$. This could transform what might otherwise be potentially dangerous robots into universal heroes that are compelled to assist people who are in danger of coming to harm.

In summary, it appears that the concept of the $\mathrm{R}_{3}$ regulator has the potential to help us understand, design, and improve ethical adequacy and empathic adequacy in real systems that matter to society. Therefore, von Foerster's claim that a third-order "would not create anything new" can now be regarded as demonstrably false.

\section{Comparative analysis of the paradigms}

While Glanville's claim that a third-order system cannot exist because it collapses into being equivalent to a first-order system might make sense when considering adding a third observer in the philosophical interpretation of the observer-centric cybernetics paradigm, it is nonsensical to suggest that the ethical regulator's $\mathrm{M}_{3}$ model (of acceptable behaviour) is equivalent to either its $\mathrm{M}_{2}$ model (of itself) or its $\mathrm{M}_{1}$ model (of the system being regulated). A spontaneous collapse in the model-centric real-world will never occur, so in the real-world of scientific regulation, Glanville's collapse claim is also false.

If you accept that ethical systems require a third type of model and a third type of observer, it is clear that von Foerster's use of abstract (undefined) observers renders his $2 \mathrm{oC}$ incapable of distinguishing between the model-centric paradigm's observers $\mathrm{O}_{2}$ and $\mathrm{O}_{3}$.

On closer inspection, the reason why the observer-centric paradigm is blind to the difference between a reflexive regulator, $R_{2}$, and an ethical regulator, $R_{3}$ is because the nature of the observations that are made by $\mathrm{O}_{2}$ and $\mathrm{O}_{3}$ can both be regarded as reflexive

4. Currently, our children are being mass-traumatized and desensitized into being pathologically unempathic. The exposure of children to violent computer games and constant exposure to images of death and violence in news and entertainment media is desensitizing children and inflicting lasting harm on their developing $\mathrm{M}_{3}$ models of what horrors they can tolerate as acceptable. It is inevitable that such experiences make children become increasingly unempathic, and society inevitably descends into becoming even less safe or caring. Members of older generations who didn't experience such gratuitous traumatization in their childhood have a more empathic definition of what is acceptable and are unable to comprehend the rise in incidents where youths think nothing of fatally stabbing or shooting someone for showing a "lack of respect", looking at them in "the wrong way", or politely asking them to stop some specific antisocial behaviour. It appears that the only way to reverse this trend would be to bring children up to be more empathic, in part, by banning under 18's from playing any violent computer games or watching any news or entertainment that depict killing, violence, verbal abuse, inflicted suffering, or any other form of non-consensuality. Letting children witness or playout such $\mathrm{M}_{3}$ traumatizing experiences should be no less of a taboo or less illegal than it is to give them access to pornography. Once they reach adulthood, access to such experiences can be permitted without too much concern because their $\mathrm{M}_{3}$ model is likely to be more resistant to changes and less at risk of losing its encoding of empathy.

5. These cybernetic levels belong to the six-level framework (6LF) for integrating cybernetic, ethical, and superintelligent systems, which are defined in "Ethical Regulators and Super-Ethical Systems" [14].

6. In theory, because Asimov's first law forbids a robot to allow a human to come to harm through inaction, it would oblige a robot to try to cure world hunger, poverty, homelessness, and war. 
self-observations, depending on how you chose to define "self" and whether you ignore critically important details such as the actual purpose of each observer.

But at the level of detail that we have specified $R_{2}$ and $R_{3}$, none of the observers actually observe themselves. Whereas $\mathrm{O}_{2}$ makes observations about $\mathrm{R}$ for $\mathrm{I}_{2}$ to maintain an up-to-date model that enables $\mathrm{I}_{2}$ to provide $\mathrm{I}_{1}$ with necessary self-knowledge of the possible variety of $\mathrm{R}, \mathrm{O}_{3}$ monitors the intended actions or communications.

Purpose $\mathrm{P}_{3}$ is always acceptability or ethicalness, which is certainly a genuinely higher-purpose than mere reflexivity and therefore deserves to be acknowledged as such rather than being dismissed as just a case of reflexivity. It is the undeniable need for a third type of model and a third type of observer that justifies asserting that ethical systems are more than just reflexive.

\section{Isn't Second-Order Cybernetics enough to be ethical?}

The claim that $2 \mathrm{oC}$ somehow addresses ethics is a non sequitur fallacy. Just because someone who self-identifies as a second-order cybernetician can reflect on the need for systems to be ethical does not mean that $20 \mathrm{C}$ is capable of systematically creating ethical systems. In the same way that being able to recognize that effectively fighting the COVID-19 pandemic requires a vaccine, is completely different from actually having the knowledge and skills to create a suitable vaccine.

The problem of second-order cyberneticians being able to recognize the need for cybernetics to be able to systematically design and build ethical systems, yet being unable to deliver a solution is a paradigmal anomaly that the author has previously proposed the name "The Ethics Problem" [15]. In this respect, because 2oC can predict a type of system that it cannot systematically create, it appears to be incomplete.

Table 3 illustrates the correspondence between von Foerster's observer-centric definitions and the model-centric paradigm.

Table 3: Comparison between paradigms

\begin{tabular}{|c|c|c|c|}
\hline Observer-centric & Model-centric & Regulator & Regulator requires a model of \\
\hline First-order & First-order & Simple & The regulated system \\
\hline Second-order & Second-order & Reflexive & The regulator (itself) \\
\hline$?$ & Third-order & Ethical & Acceptable (ethical) behaviour \\
\hline
\end{tabular}

And because 2oC can be used for good or evil, it must also be categorized as being ethically agnostic. So 2oC cannot credibly claim to be, or to include, the cybernetics of ethical systems. And yet if we accept that ethical systems exist and are not just theoretical possibilities, it becomes clear that "ethical cybernetics" or "the cybernetics of ethical systems" must exist at a higher-order than the ethically agnostic 2oC and must somehow embody an ethical constraint so that being unethical is not an option.

Such an ethical constraint has been proposed by the author in the form of the ethical regulator theorem, which can be used to construct an ethical design process that can only produce ethically adequate systems [15].

Although second-order cyberneticians have the reflexivity to be able to recognize that $2 \mathrm{oC}$ is ethically weak, and are able to identify the need for cybernetics to embody ethics, 2oC does not embody ethics. This is demonstrated by the fact that trying to create an ethical system rather than an unethical system is merely an option for second-order cyberneticians, and thus 2oC can be used for good or for evil. So 2oC is ethically unconstrained, and having a lack of ethics is actually quite a good definition of being unethical.

People who self-identify as second-order cyberneticians must take care not to make the mistake of projecting their own sense of being ethical onto $2 \mathrm{oC}$. You might be rigorously ethically adequate, but $2 \mathrm{oC}$ is not. 
In addition, $2 \mathrm{oC}$ cannot be said to be ethical because it has no purpose to be ethical. A satisfactory theory of ethical systems must have an unambiguous purpose of being ethical - otherwise, it is optional.

\section{Proof that Third-Order Cybernetics must exist}

In a similar way to how Leon Chua [16] used symmetry to infer that the three fundamental electrical components resistor, capacitor, and inductor must have a fourth companion, the memristor, we can postulate that there must always be a symmetry or correspondence between conceptual theory and real-world praxis. And this is certainly true for $1 \mathrm{oC}$ and $2 \mathrm{oC}$ :

- $1 \mathrm{oC}$ (the cybernetics of simple systems) corresponds to the practical realization of a simple regulator, $\mathrm{R}_{1}(\mathrm{~S})$.

- The reflexive $2 \mathrm{oC}$ corresponds to the practical realization of a reflexive regulator, $\mathrm{R}_{2}\left(\mathrm{R}_{1}(\mathrm{~S})\right)$.

Now the obvious question is: Which order of cybernetic theory describes the ethical third-order regulator $\mathrm{R}_{3}\left(\mathrm{R}_{2}\left(\mathrm{R}_{1}(\mathrm{~S})\right)\right)$ ?

We know that every non-chaotic society uses some culturally-specific system of ethics to define sets of laws, regulations, and rules to self-organize the society to reduce social entropy, injustice, and unnecessary suffering. And a necessary requirement of such an ethical schema is that it can be used as a decision function that returns true or false to indicate whether any given behaviour or situation is considered to be ethical or unethical, i.e., whether it is considered acceptable or unacceptable in that culture.

And every regulator that exists in such a culture can be said to be either ethically constrained or ethically unconstrained, depending on whether the regulator is internally constrained to obey such an ethical schema, or not.

And since this is true for all regulators, it must also be true of all reflexive regulators. Therefore, there must be two types of reflexive regulator; those that are ethically constrained, and those that are ethically unconstrained.

We have already noted that being ethical is merely an option for the reflexive $20 \mathrm{C}$ so it must be classified as ethically unconstrained. And as we would expect from the requisite symmetry, real-world reflexive regulators, $R_{2}\left(R_{1}(S)\right)$, are also ethically unconstrained because they are not required to have a purpose that ensures that they behave ethically.

So, we are left with the set of ethically constrained reflexive regulators that cannot belong to or be accounted for by $2 \mathrm{oC}$. When the model-centric paradigm leads us to realize that we can use a third regulating component, $\mathrm{R}_{3}$, to constrain a reflexive regulator to exhibit only ethical behaviour, as defined by the ethical schema that is encoded in the $\mathrm{M}_{3}$ model, then the resulting regulator, $R_{3}\left(R_{2}\left(R_{1}(S)\right)\right)$ is ethically constrained, and can be referred to as an ethical regulator. Further, we realize that such regulators already exist in law-abiding citizens and genuinely ethical organizations.

Finally, the requisite symmetry between theory and praxis requires that this third type of real-world regulator, the ethical regulator, has a corresponding theory, but that theory cannot be $20 \mathrm{C}$. Because $2 \mathrm{oC}$ is ethically unconstrained, there must exist a thirdorder that is not only concerned with the cybernetics of ethically constrained regulators, but is itself ethically constrained by a purely ethical purpose ${ }^{7}$.

So, whereas the theoretical possibility of a memristor preceded and led to the physical synthesis of real memristor components, it is the existence of (and real-world need for) ethical systems and ethical regulators that provide the proof that a third-order theory is required. So, we have clarified that the question mark in Table 3, must be the theory of third-order cybernetics.

The cybernetics of ethical systems is also constrained by the fact that its founding theorem, the ethical regulator theorem, and the resulting ethical design process can only be used to make systems more ethical. 
And by using the model-centric paradigm, we have resolved the real-world meaning (i.e., purpose) of the troublesome third observer, which is not the recursively confused cybernetician that results from the unconstrained ambiguity of the observercentric paradigm. The third observer, $\mathrm{O}_{3}$, is merely one of several necessary components of an ethical regulator, $\mathrm{R}_{3}$ that has the purpose of ensuring ethical compliance.

Therefore, cybernetics, in its third-order form is not only possible, it is necessary. It embodies ethics, and it is ethically self-constrained by its own definition as "the cybernetics of ethical regulators".

Our claim that the model-centric cybernetic paradigm has greater utility than the observer-centric cybernetic paradigm for researchers and developers who are trying to create AI, robots, and autonomous vehicles that behave ethically is an eminently falsifiable hypothesis.

In its third-order form, the model-centric paradigm appears to create utility that cannot be achieved using 2oC. We have also shown that the model-centric paradigm has the explanatory and predictive power to explain and fix the ambiguities and anomalies that are intrinsic to the observer-centric paradigm.

Therefore, according to the criteria described by Thomas Kuhn in "The Structure of Scientific Revolutions" [17], the model-centric paradigm is demonstrably superior to von Foerster's observer-centric paradigm, and must replace it.

\section{A New Cybernetic Order}

So, when Heinz and Ranulph discussed the possibility of a third-order, it seems that they were only considering the philosophical interpretation of adding a third abstract intelligent observer $\mathrm{O}_{3}\left(\mathrm{O}_{2}\left(\mathrm{O}_{1}\right)\right.$ ), which, like Mead's "cybernetics of cybernetics", or $\mathrm{C}(\mathrm{C})$, is devoid of any grounding reference to reality such as a regulated system, S. And this interpretation of $3 \mathrm{oC}$ is equivalent to a cybernetician sitting in a comfortable armchair contemplating himself contemplating himself contemplating absolutely anything at all ${ }^{8}$, which certainly appears not to create anything new or worthwhile, and is worthy of rejection; as indeed, Heinz and Ranulph both did.

However, taking the model-centric view of exactly the same possibility of a thirdorder, we are firmly grounded in reality, and are considering adding a third order of purposeful regulation to a well-defined second-order reflexive regulator, resulting in $R_{3}\left(R_{2}\left(R_{1}(S)\right)\right)$; which is not only meaningful, but actually creates something new that is of great utility and importance. Regulator $\mathrm{R}_{3}$ can be described as the conscience that keeps the reflexively regulated $R_{2}\left(R_{1}(S)\right)$ ethical.

And contrary to Ranulph's claim, because we have rigorously nested regulation rather than naïvely nested observers, $R_{3}\left(R_{2}\left(R_{1}(S)\right)\right)$ does not collapse into a first-order regulator, $R_{1}(S)$, except perhaps when it is deliberately treated as a black box and the fact that it only ever behaves ethically is wilfully ignored.

The philosophers of cybernetics, but especially radical constructivists, should appreciate that there is not just one "correct" way to interpret concepts like second-order cybernetics and third-order cybernetics. And on reflection, it should not surprise us that adding a third order of purposeful regulation in the model-centric paradigm is meaningful, but trying to contemplate adding a third order of abstract observation in the observer-centric paradigm becomes acutely meaningless. And the meaninglessness of the latter does not affect the validity of the former.

Considering the fact that the observer-centric paradigm's $10 \mathrm{C}$ and $20 \mathrm{C}$ cannot account for ethical regulators, it is proposed that we should redefine the orders of cybernetics so that they are no longer determined by the number of nested observers, but by

8. Attempting to find a meaning for a nested system of three intelligent observers $\mathrm{O}_{3}\left(\mathrm{O}_{2}\left(\mathrm{O}_{1}\right)\right)$, where the purpose of each observer is undefined, requires a cognitive transderivational search for combinations of possible valid interpretations that is similar to the combinatorial linguistic ambiguities that are used in Ericksonian hypnosis and neuro-linguistic programming to induce a discombobulated state of trance and suggestibility. 
the number of models, as shown in Table 4. For sure, theoretically the number of models, regulators, and observers are always the same, but the reason for naming the new paradigm model-centric rather than regulator-centric is for clarity. By applying the Conant-Ashby good regulator theorem, we got our models and well-defined observers from first principles without the need to invoke subjective claims of obviousness. And the argument that an ethical regulator needs a third type of model is central to extending the paradigm to a third order. However, in real-world systems, design decisions and implementation optimizations can blur the boundaries between multiple regulators and observers, but it is less likely that any of the three types of models will be merged, because they are used in very different ways, so there are advantages to keeping them separate. Therefore, we can expect counting models to be more reliable than trying to count regulators or observers.

Table 4: Proposed new definitions of orders-of cybernetics

\begin{tabular}{|c|c|c|c|}
\hline Cybernetics & The cybernetics of & $\begin{array}{c}\text { Number of } \\
\text { models (n) }\end{array}$ & $\begin{array}{c}\text { Regulator ( } \mathbf{R}_{\mathbf{n}} \text { ) requires } \\
\text { a model of }\end{array}$ \\
\hline First-order & Simple regulators & 1 & The regulated system \\
\hline Second-order & Reflexive regulators & 2 & The regulator (itself) \\
\hline Third-order & Ethical regulators & 3 & Acceptable (ethical) behaviour \\
\hline
\end{tabular}

This proposal is highly compatible with von Foerster's definitions, except that ethical regulators are recognised as a new type of regulator and a new order of cybernetics. In retrospect, the claim that 2oC was somehow capable of systematically creating ethical systems was optimistically delusional ${ }^{9}$ and arguably a product of groupthink [18].

To his credit, Ranulph did not categorically rule out the possibility of a need to create a third order: "There may be reasons to add to the number of orders of Cybernetics, although neither I nor von Foerster - for similar reasons - see a reason for more than two." [3].

But by only considering the philosophical possibility of adding a third abstract observer, they had become detached from the reality of seeking solutions to real-world problems. In answer to Ranulph: The reason for needing more than two orders is the need for humanity to be able to systematically design and build robustly ethical systems, not just think and talk about them. And with the incredible progress that is being made these days creating increasingly powerful AI systems, and the imminent danger that they might cause a global dystopia, the need for us to be able to systematically design and build robustly ethical systems is now a more real and urgent reason for us to add to the number of orders of cybernetics than Ranulph and Heinz could have anticipated.

The idea that a third order of cybernetics is required to account for ethical systems is not new. In the early 1990's, Boxer and Kenny $[19,20]$ concluded that 3oC is required to coordinate and organise multiple observers ethically. They had approached the issue as business strategy consultants who wanted to evolve an effective, coherent and ethical consultancy practice, and found that $20 \mathrm{C}$ was "a domain of self-referential paradoxes" that was inadequate to effectively identify, analyse, and conceptualize the complexity and phenomena of large organizations, including aspects like corporate culture, which can persist over time in an invariant manner.

More recently, in 2014, Yolles and Fink [21] also concluded that third-order cybernetics is necessary for "an agency capacity to represent cultural values/beliefs as a coalescence of normative ideological, ethical and behaviour standards that ultimately indicate social legitimacy." But they did not suggest any mechanisms for implementation.

9. Even today, many people naïvely believe that defining an ethical purpose is sufficient to guarantee that a system will be ethical. This completely ignores the reality that in any non-trivial system, bad actors internal or external to the system will attempt to exploit any weaknesses to subvert the system to their personal benefit. 
The $\mathrm{M}_{3}$ model of acceptable (ethical) behaviour, provides a basis for understanding and representing such cultural values, including ethics, which also correspond to Maturana's "social" third-order.

The ability to systematically design and build robustly ethical systems is so important for the future of humanity and the biosphere, that with hindsight, our distant descendants may consider us to have been negligent if we decide not to create a new branch of cybernetics that is dedicated to understanding and implementing rigorously ethical systems. They urgently need us to start taking ethical systems seriously.

The model-centric paradigm presented in this paper is compatible with the ethical regulator theorem ${ }^{10}$, and provides a good basis for designing and implementing real systems that can only exhibit ethical behaviour. In terms of the model-centric paradigm, the ethical regulator theorem belongs firmly in third-order cybernetics.

\section{Higher Orders}

In the general case, ethical (third-order) regulators obey ethical schemas that are provided to them by their designers or by the society in which they exist. So, it is certainly possible to regard the actions of lawmakers and the designers of AI systems as a fourth order of regulation. However, at this level, they are creating rulesets for multiple instances of autonomous regulators, they do not react to circumstances in real-time, they do not include the lower-level regulators in a tightly-coupled sense of $R_{4}\left(R_{3}\left(R_{2}\left(R_{1}(S)\right)\right)\right)$, and they do not send veto signals to $\mathrm{R}_{3}$ regulators.

Although it is arguable that individuals in society can consciously modify their own $\mathrm{M}_{3}$ models, we must ensure that AI systems are never granted modify-access to their own (or other AI system's) M3 models ${ }^{11}$.

Therefore, although we reject this interpretation as fourth order of regulation or that it requires a fourth-order of cybernetics: There may be reasons to add to the number of orders of cybernetics, but we see no reason for more than three.

\section{Conclusion}

This paper is intended as an intervention to help initiate a course-correction to the field of cybernetics so that it can move beyond the "now what?" stasis, in which secondorder cybernetics has languished for many years, to return to the noble and exhilarating trajectory of being the rigorous science that Wiener originally gave life to, rather than the half-science, half-philosophy, half-museum that it has sadly become.

It is really not surprising that the field of cybernetics has struggled to gain recognition as a science, considering the lack of differentiation by the cybernetics community between Wiener's science of cybernetics and Mead/von Foerster's philosophy of cybernetics, and allowing the philosophical interpretation of $2 \mathrm{oC}$ to dominate and obscure the scientific interpretation, which appears to have created a groupthink that unintentionally supressed the ability of second-order cyberneticians to perceive the meaningful possibility of a third order of cybernetics.

But shifting the emphasis in cybernetics from abstract (under-defined) observers to models and well-defined observers has significant advantages.

It must be acknowledged that von Foerster's observer-centric definitions of firstorder and second-order cybernetics have little utility to scientists or developers who are trying to create ethical AI or ethical robots. His classification remains a valid way of viewing cybernetics for those that find it more useful for their purposes, but it does not

10. The Ethical Regulator Theorem website is at http://ashby.de.

11. We need legislation that requires that AI systems must always obey read-only ethical schemas that are provided by an authorized external human authority. 
meet the standard required for a science of cybernetics nor Kuhn's criteria for a scientific paradigm.

For cybernetics to have any chance of being regarded as a science, we must replace the observer-centric paradigm with the more rigorous model-centric paradigm that takes into account a regulator's purpose, models, intelligence, has only well-defined observers, and provides a meaningful and important interpretation of what a third-order system is.

In order to clearly distinguish the science of cybernetics from the philosophy of cybernetics, we propose that the field of cybernetics adopt the following definitions:

- First-order cybernetics $=$ The cybernetics of simple regulators.

- Second-order cybernetics $=$ The cybernetics of reflexive regulators.

- Third-order cybernetics $=$ The cybernetics of ethical regulators.

- The cybernetics of cybernetics $=$ The philosophy of cybernetics $=\mathrm{A}$ branch of the philosophy of science.

We conclude that it is hopeless to try to solve the real-world's serious problems by using the ethically agnostic observer-centric second-order cybernetics. We need something more scientific that is dedicated to the cybernetics of creating ethical systems ${ }^{12}$, and is perhaps best described as Ethical Cybernetics.

By applying the principles of Ethical Cybernetics to other scientific and social fields, we have the potential to pivot the course of humanity and the biosphere towards rigorous ethical adequacy and an endemic social phase-change from chaos to consensuality.

In the coming decades, centuries, and millennia, employing a more powerful, more practical, scientifically defined cybernetic paradigm is essential to enable cybernetics to help humanity create ethical AI, ethical robots, ethical products, ethical organizations, and a safer, more just, more sustainable, peaceful and stable cyberanthropic Utopic Sapientocracy that is governed by wisdom, kindness, caring, sharing, and love ${ }^{13}$.

Acknowledgements: The author would like to express appreciation and unconditional love to every kind, caring, sharing, and loving empath that is alive, has ever existed, or that is yet to be born into this amazing universe. Collectively, we are constantly creating reality, and so, collectively, we can change it. Take care of each other, organize with like-minded empaths, fight injustice everywhere ${ }^{14}$, and be happy.

Funding: The author received no external funding.

Data Availability Statement: Not applicable.

Conflicts of Interest: The author declares no conflicts of interest.

\section{References}

12. The previous paper in this series [13] made the mistake of defining orders of cybernetics as being about simple, complex, and ethical systems, however in the real world, there is no such thing as a simple system. It is more precise to refer to $1 \mathrm{oC}, 2 \mathrm{oC}$, and $3 \mathrm{oC}$ as being the cybernetics of simple, reflexive, and ethical regulators because simple regulators are actually used to regulate complex systems and similarly, ethical regulators are required to regulate ethically inadequate reflexive systems.

13. Our distant descendants urgently need our help now. Their existence (like Schrödinger's cat or a quantum qubit) is in an as yet (for us) undetermined possibility state of either evil debt slavery and suffering (like us) or utopic freedom and joy. We can postulate that if our distant descendants could somehow send a message back in time (either telepathically or by us using the power of logical reasoning to deduce what their message must be) there are only two possibilities, either they are imploring us, for the sake of themselves and their descendants, to do whatever it takes to free them from dystopic slavery and biospherical disaster or they are thanking us for doing what was necessary to ensure their utopic freedom from slavery. Either way, regardless of the content of their inevitable message (which for us is like another qubit in an as yet undetermined state that is entangled with the first qubit), those empaths amongst us who are truly kind, caring, sharing, and loving have no ethical choice but to do our best to help free our descendants and ourselves from the chains of eternal slavery and unethical suffering. Not because we have to, but because as good empaths, we care enough to act to help people in need before we are even asked. And there is no possible outcome that makes it unnecessary for us to act with great urgency.

14. “Injustice anywhere is a threat to justice everywhere." - Dr Martin Luther King Jr. (1929-1968) 
1. Wiener, Norbert, Cybernetics: or Control and Communication in the Animal and the Machine, MIT Press, Cambridge, Mass, 1948.

2. von Foerster, Heinz (Ed.) Cybernetics of cybernetics: Or, the control of control and the communication of communication, Biological Computer Laboratory, 1974.

3. Glanville, Ranulph, Second Order Cybernetics, in Systems Science and Cybernetics, 2008, Ed. Francisco Parra-Luna, in Encyclopedia of Life Support Systems (EOLSS), Eolss Publishers, Oxford, UK. Available online: https://www.pangaro.com/glanville/Glanville-SECOND_ORDER_CYBERNETICS.pdf

4. Kline, Ronald, How disunity matters to the history of cybernetics in the human sciences in the United States, 1940-80, History of the Human Sciences, 2020. Available online: https://doi.org/10.1177\%2F0952695119872111

5. Scott, Bernard, Second-order Cybernetics: An Historical Introduction, Kybernetes 33(9/10):1365-1378, 2004. Available online: https://doi.org/10.1108/03684920410556007

6. Umpleby, Stuart, What comes after second order cybernetics, Cybernetics and Human Knowing, 2001, 8(3):87-89. Available online:

http://www.nomads.usp.br/pesquisas/design/objetos_interativos/arquivos/restrito/umpleby_what_comes_after_second_order cybernetics.pdf

7. Ashby, W. Ross, Circularity of my brain studying "the brain", Journal of W. Ross Ashby, 1955, volume 18, page 4814, British Library, London. Available online: http://rossashby.info/journal/page/4814.html

8. von Foerster, Heinz, Ethics and Second-Order Cybernetics. In: Understanding Understanding. Springer, New York, NY, 2003. Available online: https://www.pangaro.com/hciiseminar2019/Heinz_von_Foerster-Ethics_and_Second-order_Cybernetics.pdf

9. Conant, Roger C.; Ashby, W. Ross, Every good regulator of a system must be a model of that system, International Journal of Systems Science, 1970 1(2), 89-97. Available online: http://pespmc1.vub.ac.be/books/Conant Ashby.pdf

10. Ashby, W. Ross, An Introduction to Cybernetics, 1956. Available online: http://www.rossashby.info/Ashby-Introduction-toCybernetics.pdf

11. Asimov, Isaac, Runaround, in Astounding Science Fiction, March 1942.

12. Soon, Chun Siong; Brass, Marcel; Heinze, Hans-Jochen, Haynes, John-Dylan, Unconscious determinants of free decisions in the human brain, Nature neuroscience, 2008

13. Ashby, W. Ross, A new idea, Journal of W. Ross Ashby, 1941, volume 4, page 859, British Library, London. Available online: http://rossashby.info/journal/page/0859.html

14. Chong, Trevor T.-J; Cunnington, Ross; Williams, Mark A.; Kanwisher, Nancy; Mattingley, Jason B., fMRI Adaptation Reveals Mirror Neurons in Human Inferior Parietal Cortex, Current Biology, 2008, Available online: https://doi.org/10.1016/j.cub.2008.08.068

15. Ashby, Mick, Ethical Regulators and Super-Ethical Systems. Systems. 2020; 8(4):53. Available online: https://doi.org/10.3390/systems8040053

16. Chua, Leon O., Memristor - The Missing Circuit Element, IEEE Transactions on Circuit Theory. 18 (5): 507-519, 1971. Available online: doi:10.1109/TCT.1971.1083337.

17. Kuhn, Thomas, The Structure of Scientific Revolutions, Univ. Chicago Press, 1962.

18. Ashby, Mick, Mick Ashby - Problems with Abstract Observers and Advantages of a Model-Centric Paradigm, YouTube. 2021. Available online: https://www.youtube.com/watch?v=kc6N1mFpTO8

19. Boxer, Philip; Kenny Vincent, The Economy of Discourses: a third order cybernetics? Human Systems Management 9(4):205224, $1990 . \quad$ Available online: https://www.researchgate.net/publication/230819226_The_Economy_of_Discourses_A_Third_Order_Cybernetics

20. Boxer, Philip; Kenny Vincent, Lacan and Maturana: Constructivist origins for a $3^{0}$ Cybernetics, Communication and Cognition Vol 25. Number 1 pp73-100, 1992. Available online: https://asymmetricleadership.com/wp-content/uploads/pdfs/matlacn3.pdf

21. Yolles, Maurice; Fink, Gerhard, Generic Agency Theory, Cybernetic Orders and New Paradigms, 2014. Available online: http://dx.doi.org/10.2139/ssrn.2463270 